\title{
Magnetoresistance (MR) of twisted bilayer graphene on electron transparent substrate
}

Sung Ju Hong ${ }^{\mathrm{a}}$, Julio A. Rodríguez-Manzo ${ }^{\mathrm{b}}$, Kyung Ho Kimª, Min Park ${ }^{\mathrm{a}}$, Seung Jae Baek ${ }^{\mathrm{a}}$, Dmitry I. Kholin ${ }^{\mathrm{c}}$, Minwoo Lee ${ }^{\mathrm{d}}$, Eun Sang Choi ${ }^{\mathrm{e}}$, Dae Hong Jeong ${ }^{\mathrm{d}}$, Dawn A. Bonnell ${ }^{\mathrm{f}}$, Eugene J. Mele ${ }^{\mathrm{b}}$, Marija Drndic ${ }^{\mathrm{b}}$, A. T. Charlie Johnson ${ }^{\mathrm{b}, \mathrm{f}^{*}}$ and Yung Woo Park ${ }^{\mathrm{a}, * *}$

${ }^{a}$ Department of Physics and Astronomy, Seoul National University, Seoul 151-747, Korea

${ }^{b}$ Department of Physics and Astronomy, University of Pennsylvania, Philadelphia, Pennsylvania 19104, USA

${ }^{c}$ P. L. Kapitza Institute for Physical Problems, Russian Academy of Sciences, ul. Kosygina 2, Moscow 119334, Russia

${ }^{\mathrm{d}}$ Department of Chemistry Education, Seoul National University, Seoul 151-742, Korea

${ }^{e}$ National High Magnetic Field Laboratory, Tallahassee, Florida 32310, USA

${ }^{\mathrm{f}}$ Nano-Bio Interface Center, University of Pennsylvania, Philadelphia, Pennsylvania 19104, USA

E-mail:cjohnson@physics.upenn.edu, ywpark@phya.snu.ac.kr 
We studied the magnetoresistance (MR) of twisted bilayer graphene (tBLG) on electron transparent substrate. Samples of tBLG were assembled on free-standing silicon nitride $\left(\mathrm{SiN}_{\mathrm{x}}\right)$ membranes $(<100 \mathrm{~nm}$ thick) by transferring chemical vapor deposition (CVD)-grown single layer graphene (SLG) twice; this allowed the measurement of the angle of rotation between the two layers, the twist angle, by electron diffraction using a transmission electron microscope (TEM). To compare with the previous reports on tBLG, we performed Raman spectroscopy on our samples. We measured the MR of tBLG for two different twist angles: $2^{\circ}$ (small) and $18^{\circ}$ (large). The MR showed superposition of two Shubnikov de Haas $(\mathrm{SdH})$ oscillations for both angles. An analysis of the oscillation peaks by Landau fan diagrams showed difference as twist angle. While the large twist angle $\left(18^{\circ}\right)$ sample had two anomalous $\pi$ Berry's phases, the small twist angle $\left(2^{\circ}\right)$ sample had conventional $2 \pi$ and anomalous $\pi$ Berry's phase depending on carrier density.

Keywords : Twisted bilayer graphene, Magnetoresistance, Landau fan diagram, Berry's phase 


\section{Introduction}

The low energy electronic properties of SLG are described by a model for massless Dirac fermions and contain unusual physics including an anomalous quantization of the Hall conductance, Klein tunneling [1-4] and other electronic phenomena that have been studied in transport [1-6] and optics [7] and may be useful for applications [8-10]. In multilayer graphene this low energy physics is changed by the development of electronic coherence between the layers. This turns out to be exquisitely sensitive to the atomic registry between the neighboring layers and their stacking order, and introduces a new degree of freedom for controlling the low energy physics. The possibility of exploiting these effects in various multilayer structures is being vigorously pursued. tBLG are systems where the crystallographic axes are rotationally misaligned and provide a particularly interesting family of materials because the interaction between its layers is determined by the relative twist angle and leading low energy physics that is unexpectedly rich [11-14].

The rotational misorientation of graphene layers in $\mathrm{tBLG}$ induces a mismatch of the momenta of the Dirac points of the two layers. Band calculations suggest that this produces a rotation angledependent renormalization of the Fermi velocity at low energies and a new van Hove singularity (vHs) in the density of states due to the hybridization of the Dirac cones at an energy scale where they merge. These features are observed in STM experiments [15-18]. Raman spectroscopy and electron diffraction are also sensitive to twist angle in tBLG [19-26]. In particular, two recent studies [21,22] of Raman spectroscopy dependence on twist angle (confirmed by electron diffraction) show the correlation between Raman scattering and electronic band structure for different angles of rotation. The gate dependent resistance of tBLG was reported [27-33], although the twist angles between the two graphene layers were not determined, assuming that the two independent graphene layers are stacked together. They analyzed the date with two carrier densities of bottom and top layer in tBLG. On the other hand, there are theoretical predictions $[12,14]$ suggesting the existence of Moiré butterfly pattern when the twist angle, $\theta$, becomes small (less than $4^{\circ}$ ). It is explained as due to the strong coupling between the two layers at small twist angle. Moreover, experimental observations for Hofstadter butterfly are recently reported in SLG or bilayer graphene (BLG) on h-BN substrate where graphene and h-BN layers are slightly mismatched less than $2^{\circ}[34-36]$.

In this paper, we report the MR of tBLG samples in which the twist angle was directly measured with electron diffraction. We used an electron transparent $\mathrm{SiN}_{\mathrm{x}}$ membranes $(<100 \mathrm{~nm}$ thick) as the substrate to acquire electron diffraction patterns and measure the transport properties of the same sample [37]. We observed superposition of two oscillations in both measured angles. We constructed Landau level fan diagrams for each to identify the Berry's phase for the two samples and find that they are different, depending on twist angle. While two anomalous $\pi$ Berry's phases are observed for the large twist angle $\left(18^{\circ}\right)$ sample, conventional $2 \pi$ and anomalous $\pi$ Berry's 
phase are obtained for the small twist angle $\left(2^{\circ}\right)$ sample.

\section{Experimental}

tBLG samples were made by transferring SLG twice. SLG was grown on a $\mathrm{Cu}$ foil (Sigma Aldrich, $25 \mu \mathrm{m}$ thick and $99.98 \%$ purity) at $1050{ }^{\circ} \mathrm{C}$ at atmospheric pressure, with a mixture of $\mathrm{Ar}, \mathrm{H}_{2}$ and $\mathrm{CH}_{4}$ gases [38]. We used a standard wet etching method to transfer SLG $(0.1 \mathrm{M}$ ammonium persulfate solution) [39]. To check the mobility, we did separate experiment for another SLG sample on $\mathrm{SiO}_{2}(300 \mathrm{~nm})$ with back gate. And we obtained the mobility of 1625 $\mathrm{cm}^{2} /$ Vs near Dirac point $\left(\mathrm{V}_{\text {Dirac }}=40 \mathrm{~V}\right)$ at $77 \mathrm{~K}$.

The tBLG device fabrication in Fig. 1(a) is described as follows. (i) $\operatorname{SiN}_{x}(100 \mathrm{~nm}) / \mathrm{Si}(500$ $\mu \mathrm{m}) / \mathrm{SiN}_{\mathrm{x}}(100 \mathrm{~nm})$ wafer is prepared. (ii) One side of the wafer was first patterned with photolithography and later etched with a $\mathrm{SF}_{6}$ plasma treatment. (iii) Afterwards, anisotropic wet etching of $\mathrm{Si}(100)$ in a $\mathrm{KOH}$ solution was performed to obtain the $\mathrm{SiN}_{\mathrm{x}}$ membrane. After transferring SLG twice, (iv), we isolated tBLG channels by using a negative tone resist (HSQ) and $\mathrm{O}_{2}$ plasma etching. (v) Large contact pads were fabricated by combining photo and e-beam lithography, followed by thermal evaporation of $\mathrm{Cr} / \mathrm{Au}(5 / 100 \mathrm{~nm})$ and small contact pads of $\mathrm{Cr} / \mathrm{Au}(5 / 150 \mathrm{~nm})$ were made to connect the tBLG channels. Finally, we obtained $1 \mu \mathrm{m} \times 0.5 \mu \mathrm{m}$ size of tBLG channel and measured MR, electron diffraction and Raman spectroscopy on the same sample.

Fig. 1(b) shows an optical (left) and TEM (right) images of a fabricated tBLG device on a $\operatorname{SiN}_{\mathrm{x}}$ membrane. In the optical image, the light blue square region is the free-standing $\operatorname{SiN}_{\mathrm{x}}$ membrane and the dark region surrounding the square is the $\mathrm{SiN}_{\mathrm{x}} / \mathrm{Si}$ substrate. The TEM image is obtained from red dashed box in optical image. We describe two-probe measurement scheme and guide the tBLG channel by blue rectangle.

We measured the MR with two probes using a standard lock-in technique at low temperature, and high magnetic fields (the standard $35 \mathrm{~T}$ DC resistive magnet in the NHMFL in Tallahassee, Florida, U.S.A.). Raman spectroscopy and electron diffraction were performed after the electrical transport measurement. The Raman signal (532 nm wavelength) was enhanced by adding a layer of $40 \mathrm{~nm} \mathrm{Pd}$ underneath the $\mathrm{SiN}_{\mathrm{x}}$ membrane after finishing electron diffraction experiment. TEM analysis and electron diffraction were performed in a JEOL 2010 microscope, with an acceleration voltage of $200 \mathrm{kV}$. We acquired electron diffraction patterns from areas of $630 \mathrm{~nm}$ in diameter by using a selected area diffraction pattern (SAED) aperture.

\section{Result and discussion}




\subsection{Raman spectroscopy and electron diffraction experiment}

Raman spectroscopy strongly depends on the interlayer coupling in bilayer graphene (BLG). For decoupled (or weakly coupled) tBLG, the $2 D$ peak shows high intensity, a narrow width and a blue shift $[19,21]$. A strong $G$ peak enhancement is also observed near rotation angles where the energy scale of vHs becomes similar with that of the excitation laser [20-22]. First, we show raw Raman spectroscopy result in Fig. 2(a). We observed representative Raman peaks, for instance, $D, G, 2 D$ peaks. In order to analyze $G$ and $2 D$ peaks in detail, we subtracted base signal which comes from substrate background. Fig. 2(b) shows $G$ and $2 D$ peaks of the two tBLGs after subtracting background. The $2 D$ peak positions for $2^{\circ}$ and $18^{\circ}$ are blue shifted to $2695 \mathrm{~cm}^{-1}$ and $2694 \mathrm{~cm}^{-1}$, respectively. This blue shift is comparable with previous Raman results [21]. The full width half maximum (FWHM) for the $2 D$ peak of $2^{\circ}, 42 \mathrm{~cm}^{-1}$, is narrower than the expected value of $60 \sim 70 \mathrm{~cm}^{-1}$. The $2 D \mathrm{FWHM}$ of $18^{\circ}, 38 \mathrm{~cm}^{-1}$, however, agrees with previous reported values. Since it is difficult to consider the $2 D$ signal as definite evidence of the rotation angle dependence, we also analyzed the $G$ peak. The integrated intensity of the $G$ peak for $18^{\circ}$ is twice as large as the one for $2^{\circ}$. This difference can be interpreted by a singularity of joint density of state (JDOS) which is reported near $12.5^{\circ}$ in a previous study with same excitation energy of laser $(2.33 \mathrm{eV})$ [22]. The $D^{\prime}$ peak known to be accompanied with large $D$ peak is observed around $1620 \mathrm{~cm}-1$ for both $2^{\circ}$ and $18^{\circ}$, which are in agreement with edge effects in our narrow channel widths $(500 \mathrm{~nm})$.

Electron diffraction patterns easily indicate the twist angle. For example, Fig. 2(c) and (d) show the diffraction patterns for tBLG with twist angles of $2^{\circ}$ and $18^{\circ}$, respectively. In Fig. 2(c), white dashed circle is enlarged to inset of Fig. 2(c). Two arrows indicate twist angle of $2^{\circ}$ between two dots. We also obtain twist angle of $18^{\circ}$ between red and yellow dashed lines in Fig. 2(d). The patterns show two sets of spots with hexagonal symmetry rotated by the proper amount, as expected. The existence of only two sets means that the tBLG channels consisted of two singlegrain SLGs; otherwise more sets of patterns would have been observed [40]. The background ring patterns result from the amorphous $\mathrm{SiN}_{\mathrm{x}}$ membrane.

\subsection{Magnetoresistance (MR)}

We present MR of $2^{\circ}$ and $18^{\circ}$ tBLG at several low temperatures in Fig. 3 and 4, respectively. Fig. 3(a) and 4(a) are raw data of R vs $B$ of $2^{\circ}$ and $18^{\circ}$ tBLG, respectively. Even though our data does not show quantum Hall state like in the reported sample of moderate mobility [41], we observed that there is an oscillation behavior superimposed in the increasing background signal

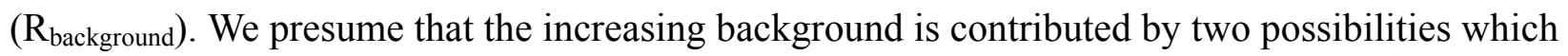
are the Hall signal proportional to $B$ in two-terminal device configuration and the $B^{2}$ behavior 
observed in ordinary metal or semiconductor. Indeed, similar behaviors were previously reported in various papers [27,28,42-44]. In addition, we found that two different oscillations are superposed in the MR plot. We called the two different frequencies as the slow and the fast oscillation, respectively. The characterization details will be discussed in the Landau fan diagram below. The two oscillations have different aspect of temperature dependent MR. While the slow oscillation did not show distinctive temperature dependence in the range from $2.8 \mathrm{~K}$ to $10.7 \mathrm{~K}$, the fast oscillation of $2^{\circ}$ and $18^{\circ}$ tBLG show temperature dependence as shown in the insets of Fig. 3(a) and 4(a) enlarged in $10<\mathrm{B}<18 \mathrm{~T}$ and $27<\mathrm{B}<35 \mathrm{~T}$ regions, respectively. That is, the amplitude of oscillation decreases as temperature increases, which is a representative behavior in Shubnikov de Haas $(\mathrm{SdH})$ oscillation. Our observation of rapid reduction of amplitude for the fast oscillation is consistent with the previous study that amplitude of our $\mathrm{SdH}$ oscillations is more rapidly reduced in high carrier density [1]. The $\Delta \mathrm{R}\left(=\mathrm{R}-\mathrm{R}_{\text {background }}\right)$ shown in Fig. 3(b) and 4(b) were obtained by subtracting background signal which is fitted by a polynomial function up to the second order in $B$. We observed that the $\Delta \mathrm{R}$ data follow the normal $\mathrm{SdH}$ oscillation behavior, that is, the amplitude of $\mathrm{SdH}$ oscillation increases as magnetic field increases. Likewise the MR plots, we found that two different oscillations are superposed in a Landau fan diagram plotting the index of the highest occupied Landau level (LL) as a function of $1 / B$. Slow SdH oscillations correspond to the right axis, $\Delta \mathrm{R}$, which are shown in the Fig. 3(c) and 4(c) for each twist angle. To distinguish the fast oscillation, we performed smoothing process and subtracted the background which is the slow oscillation part in the upper inset of Fig. 3(c) and 4(c), respectively. As a result, the Landau fan diagrams for fast oscillations are obtained as shown in Fig. 3(d) and 4(d). The local resistance minimum (maximum) corresponds to the $n(n+1 / 2)$-th LL which is presented in the left axis of panels (c) and (d) of Fig. 3 and 4 . The slope in the Landau fan diagram is the frequency of oscillation, $f_{S d H}$, which is related to the carrier density as $n_{S d H}=g(e / h) f_{S d H}$, where $g=4$ means spin and valley degeneracy, $e$ is the unit charge quantity and $h$ is Planck constant $[1,2]$. The intercept in the Landau fan diagram is known to be related with Berry's phase of carrier, for example, SLG and Bernal stacked BLG have intercept values of 0.5 and 0 in the Landau fan diagram, respectively, which means Berry's phases of $\pi$ and $2 \pi$ [45]. In two-terminal device, the quantum oscillatory phase is affected by device aspect ratio, $\mathrm{L} / \mathrm{W}$, where $\mathrm{L}$ is length and $\mathrm{W}$ is width of sample [46,47]. According to the references, the conductance, $\mathrm{G}$, has maxima (minima) for $\mathrm{L}>\mathrm{W}(\mathrm{L}<\mathrm{W})$ at incompressible densities, filling factors $\pm 2, \pm 6, \pm 10$, etc. With $\mathrm{L} / \mathrm{W}>1$ aspect ratio of our device, our Landau fan diagram analysis is valid, where the local resistance, $\mathrm{R}=1 / \mathrm{G}$, shows local minimum at LLs (i.e. local maxima in G). It is difficult to identify the geometric factor of graphene on $\mathrm{SiN}_{\mathrm{x}}$ from Fig. 1(b). However, the electron beam mask of $1 \mu \mathrm{m} \times 0.5 \mu \mathrm{m}$ size covers the graphene to prevent it from being etched in the process of fabrication. Thus, after successful fabrication the graphene has the geometry of the mask, which is $\mathrm{L}=1 \mu \mathrm{m}$ and $\mathrm{W}=0.5 \mu \mathrm{m}$. Therefore, the graphene in our device has aspect ratio of $\mathrm{L} / \mathrm{W}=2$ and the phase of a SLG is determined as $\pi$ following the analysis in the ref. 46 and 47 . It should be noted that the LL indexing is carried out by adopting local 
minimum and maximum point. Dissimilar with analysis by gate dependent MR or quantum Hall plateau, our electron transparent device configuration cause a difficulty indexing the LL. The LL indexing was based on two constrains which are (i) the adjacent local minimum and maximum have difference of 0.5 and (ii) the intercept value has to be either 0 or 0.5 in two-dimensional carbon system, respectively. From the consistency between the slopes of Landau fan diagram and the resonance peaks of Fourier transform, the LL indexing turns out to be valid.

Fig. 3(c) shows the Landau fan diagram for the small twist angle $2^{\circ}$. The $\Delta \mathrm{R}$ vs $1 / B$ plot in Fig. 3(c) shows two different oscillations. Upper inset of Fig. 3(c) shows the fast oscillation enlarged in the region of red dashed box, which is superimposed with the slow oscillation. This superposition of the two oscillations is shown with the two resonance peaks in the lower inset of Fig. 3(c) which is the Fourier transform of $\Delta \mathrm{R}$ vs $1 / B$ plot. We found that two resonance frequencies of 22.8 and $100 \mathrm{~T}$ corresponding to carrier densities of $2.2 \times 10^{12} \mathrm{~cm}^{-2}$ and $9.7 \times 10^{12}$ $\mathrm{cm}^{-2}$, respectively. Compared with low frequency, high frequency peak is small but distinguishable enough. Furthermore, the high frequency peak is in good agreement with the value from the slope of Landau fan diagram in Fig. 3(d). With the consistency, we conclude that the fast oscillation is not universal conductance fluctuation (UCF) which requires aperiodic fluctuation. To distinguish the fast oscillation clearly, we subtracted the slow oscillation part in the upper inset of Fig. 3(c) and plotted in Fig. 3(d). The frequencies can be also extracted from the slopes which are obtained by linear fitting in the Landau fan diagram. The linear fittings in Fig. 3(c) and (d) yield oscillation frequencies of 22.9 and $99.1 \mathrm{~T}$, respectively. These values are close to the frequencies which are directly obtained from the Fourier transform of the magnetooscillation shown in the lower inset of Fig. 3(c). Likewise, intercept values are extracted from the linear fitting in the Landau fan diagram are $0.74 \pm 0.07$ and $-0.17 \pm 0.25$ presented with red color in Fig. 5. The intercept values are similar with that of SLG in the low carrier density regime and that of BLG in the high carrier density regime, respectively. According to theoretical calculations $[48,49]$, the field dependence of the LLs is similar with that of SLG, $E_{L L} \propto \sqrt{B}$, which includes layer degeneracy below vHs energy. Above vHs, the layer degeneracy is lifted and the LL spacing is similar to that of Bernal stacked BLG where there is a quadratic dispersion relation, $E_{L L} \propto B$. The theoretical calculations estimate the energy scale for $\mathrm{vHs}, 0.1 \mathrm{eV}$, for $3.27^{\circ}$ twist angle. Using the Fermi level and carrier density relation of SLG, $E_{F}=\hbar v_{F} \sqrt{\pi n}$, where $\hbar$ is the Planck constant over $2 \pi, v_{F}$ is the Fermi velocity, and $n$ is carrier density, the observed two different frequencies (i. e. carrier densities) correspond to placing the Fermi levels at 0.17 and $0.36 \mathrm{eV}$ above the Dirac points of the two layers in our $2^{\circ}$ sample. Although the estimated 0.17 $\mathrm{eV}$ is actually larger than theoretically predicted value of the vHs scale $(0.1 \mathrm{eV})$, we see from the field dependence that the lower carrier density shows $\pi$ Berry's phase and the higher carrier density $(0.36 \mathrm{eV})$ has $2 \pi$ Berry's phase.

The $\Delta \mathrm{R}$ vs $1 / B$ plot of the $18^{\circ}$ twisted case also shows superposition of two different oscillations 
as in Fig. 4(c). Similarly, upper inset of Fig. 4(c) indicates the fast oscillation which is superimposed with the slow oscillation. As the Fourier transform in lower inset of Fig. 4(c), we extracted resonance frequencies of 18.5 and $330 \mathrm{~T}$ corresponding to carrier densities (Fermi level) of $1.8 \times 10^{12} \mathrm{~cm}^{-2}(0.16 \mathrm{eV})$ and $3.2 \times 10^{13} \mathrm{~cm}^{-2}(0.66 \mathrm{eV})$, respectively. To distinguish the fast oscillation clearly, we subtracted the slow oscillation part in the upper inset of Fig. 4(c) and plotted in Fig. 4(d). From the slopes in the Landau fan diagram shown in Fig. 4(c) and (d), the frequencies are 18.6 and $324 \mathrm{~T}$, which are consistent with the values obtained from the Fourier transform. The intercept values are $0.33 \pm 0.08$ and $0.41 \pm 0.36$, respectively, and are shown in black color in Fig. 5. Both values are near 0.5 for the large twist angle, which means that both carriers show SLG characteristic. This can be interpreted as resulting from two graphene sheets that are electronically decoupled (nearly independent) at large twist angle. Note that we also observe a deviation from the linear fitting in the Landau fan diagram in the high carrier density region as shown in Fig. 4(d). In ref. 5, the authors related to the confinement effect with the narrow channel geometry in SLG. The deviation occurs in low magnetic fields, when $2 R_{c}>\mathrm{W}$, where $2 R_{c}=4 n / k_{F}$, and $n$ is the LL index for graphene. In Fig. 4(d), the deviation starts from $n=$ 20 and yields an effective width of $W^{*} \approx 260 \mathrm{~nm}$, which is in the same order of our sample width, $500 \mathrm{~nm}$. In terms of mean free path $\left(l^{*}\right)$, we obtained $2.3 \mathrm{~nm}$ of $l^{*}$ in our sample. Since the confinement effect is valid for $l^{*}>W^{*}$, the deviation in our case is not explained by confinement effect. This could be due to the disorderness of our sample. However, we focus on the linear part in the Landau fan diagram in Fig. 4(d) which yields the fast carrier density and the value is consistent with that from the Fourier transform.

A striking observation in our data is the presence of two carrier pockets for both large and small angle tBLG, which indicates that a vertical potential difference $\approx 0.3 \mathrm{eV}$ exists between the layers. Furthermore for the sample with a small twist angle the data suggest the coexistence at the same energy of conventional (multiple of $2 \pi$ ) and anomalous $(\pi)$ Berry's phases for the high density and low density pockets, respectively. This result is distinguished from the situation inferred from measurement of the quantum Hall plateaus which indicate that a crossover from anomalous to conventional LL ordering occurs at a crossover energy scale separating the decoupled layer regime (low energy) from the vertically coupled layer regime (high energy). The data can be understood in terms of a model that describes a competition between two important energy scales in this problem. The first is the interlayer crossover scale $\Delta_{\theta}=4 \pi \hbar v_{F} \sin \left(\frac{\theta}{2}\right) / 3 a$, where $\hbar$ is the Planck constant over $2 \pi, v_{F}$ is the Fermi velocity, $\theta$ is twist angle, and $a$ is lattice parameter $(0.246 \mathrm{~nm})$ in graphene where the two Dirac cones of the two layers first merge. The second is the layer asymmetry potential $\Delta_{\mathrm{z}}$ that is responsible for a charge disproportionation between the layers. For large twist angle $\left(\Delta_{\theta}>>\Delta_{z}\right)$, the tBLG has the MR signature of two decoupled graphene sheets at low energy regime. This is the situation envisioned by the published theories for the quantum Hall effect in tBLG. However, for small twist angle $\left(\Delta_{\mathrm{z}}>\Delta_{\theta}\right)$, this has a different signature in the MR as we show below. 
The basic ideas can be understood by analyzing the band plots shown in Fig. 6. In panels (a)-(c) of Fig. 6, the band plot shows $\Delta_{\theta} \gg \Delta_{z}$ and the intercepts along the $q_{y}$ axis at \pm 5 are the rotationally offset Dirac cones. While panel (a) considers just momentum offset, panel (b) shows the effect of turning on, in succession, the interlayer potential $\Delta_{z}$ (dashed red curve) and the interlayer coupling with strength $\Gamma$ (solid black curve). The interlayer potential shifts the two Dirac points upwards and downward in energy. And the interlayer coupling produces an avoided crossing at the energy where the two Dirac cones merge. The interlayer scale in this problem is very large $\left(\approx 1.5 \mathrm{eV}\right.$ for $\left.\theta \approx 20^{\circ}\right)$ and so over a wide range of chemical potential the Fermi surfaces (lines) are loops that separately encircle the two Dirac points. For example, Fermi level depicted by blue dashed line in Fig. 6(b) correspond black circles in Fig. 6(c). This produces two carrier pockets with an anomalous intercept $(0.5)$ in the Landau level fan diagrams. If the rotation angle $\theta$ is small and the chemical potential places in the interlayer coupling gap, the system reverts to a single carrier pocket with conventional magneto-oscillations in the transport. Qualitatively this occurs because the $\pi$ Berry's phases from the two singularities add to give a total Berry phase $2 \pi$ and conventional Landau quantization. This has been examined in two previous theoretical studies. In this regime the interlayer potential $\Delta_{\mathrm{z}}$ is negligible.

Now consider the situation for very small twist angle where $\Delta_{z}>\Delta_{\theta}$, as shown in panels (d)-(f) in Fig. 6. Panel (d) considers just the effect of the momentum offset with small $\Delta_{\theta}$. When the interlayer potential is turned on (e), it becomes the dominant scale of the problem. The intersections at positive and negative energy are the electrostatically displaced Dirac points of the two layers. Turning on $\Gamma$ gives an avoided crossing near $E=0$ (this is the direct interlayer mixing) and opens up a gap for these displaced points due to second order mixing through $\Gamma$ (For the conventional model of the interlayer potential that we are considering, one of the Dirac states does not couple to the other layer and remains at the electrostatically displaced Dirac energy, the other one is split away from this energy). For chemical potential $\mu$ comparable to (a)-(c) in Fig. 6, the Fermi lines consist of two closed contours, one of which resides in the "third" band the other in the "fourth". The latter case resides entirely in a band that encircles a single Dirac point and has an anomalous Berry's phase $\pi$. The other lies in the third band and has a Berry's phase $2 \pi$ just as in the high energy regime for large twist angles (The band has exactly the same topological structure).

\section{Conclusion}

In conclusion, we measured the MR of tBLG samples with twist angles directly measured from the electron diffraction. This was possible because electron transparent $\mathrm{SiN}_{\mathrm{x}}$ membranes were used as substrates. The MR showed superposition of two SdH oscillations in both cases, but 
Landau fan diagram analysis showed that the carrier types are different for small and large twist angles. For the $18^{\circ}$, the tBLG showed two anomalous $\pi$ Berry's phases, as in SLG. In contrast, the $2^{\circ}$ tBLG sample showed both anomalous $\pi$ and conventional $2 \pi$ Berry's phase, depending on carrier density.

\section{ACKNOWLEDGMENTS}

This work was supported by the Swedish-Korean Basic Research Cooperative Program (No. 2014R1A2A1A12067266) of the NRF and the FPRD of BK21 through the MEST, Korea and NSF NBIC grant, U.S.A. M.D. and J.A.R.-M. acknowledge funding from the NIH grant R21HG00476. M.L. and D.H.J. acknowledge the support of the Pioneer Research Center Program through the NRF of Korea, funded by the Ministry of Science, ICT \& Future Planning (No. NRF-2011-0027888). This research was partially supported by National Science Foundation grant DMR-0923245, U.S.A. The high field MR was measured at the NHMFL in Tallahassee, Florida, U.S.A. 


\section{References}

[1] K. S. Novoselov, A. K. Geim, S. V. Morozov, D. Jiang, M. I. Katsnelson, I. V. Grigorieva, S. V. Dubonos, A. A. Firsov, Nature 438 (2005) 197.

[2] Y. Zhang, Y. W. Tan, H. L. Stormer, P. Kim, Nature 438 (2005) 201.

[3] M. I. Katsnelson, K. S. Novoselov, A. K. Geim, Nature Phys. 2 (2006) 620.

[4] A. F. Young, P. Kim, Nature Phys. 5 (2009) 222.

[5] C. Berger, Z. Song, X. Li, X. Wu, N. Brown, C. Naud, D. Mayou, T. Li, J. Hass, A. N. Marchenkov, E. H. Conrad, P. N. First, W. A. de Heer, Science 312 (2006) 1191.

[6] E. H. Hwang, S. Adam, S. Das Sarma, Phys. Rev. Lett. 98 (2007) 186806.

[7] R. R. Nair, P. Blake, A. N. Grigorenko, K. S. Novoselov, T. J. Booth, T. Stauber, N. M. R. Peres, A. K. Geim, Science 320 (2008) 1308.

[8] S. Bae, H. Kim, Y. Lee, X. Xu, J. -S. Park, Y. Zheng, J. Balakrishnan, T. Lei, H. R. Kim, Y. I. Song, Y. -J. Kim, K. S. Kim, B. Özyilmaz, J. -H. Ahn, B. H. Hong, S. Iijima, Nature Nanotechnol. 5 (2010) 574.

[9] Y. M. Lin, C. Dimitrakopoulos, K. A. Jenkins, D. B. Farmer, H. Y. Chiu, A. Grill, Ph. Avouris, Science 327 (2010) 662.

[10] H. Yang, J. Heo, S. Park, H. J. Song, D. H. Seo, K. E. Byun, P. Kim, I. Yoo, H. J. Chung, K. Kim, Science 336 (2012) 1140.

[11] J. M. B. Lopes dos Santos, N. M. R. Peres, A. H. Castro Neto, Phys. Rev. Lett. 99 (2007) 256802.

[12] R. Bistritzer, A. H. MacDonald, Proc. Natl. Acad. Sci. U.S.A. 108 (2011) 12233.

[13] E. J. Mele, Phys. Rev. B 81 (2010) 161405(R).

[14] P. Moon, M. Koshino, Phys. Rev. B 85 (2012) 195458.

[15] G. Li, A. Luican, J. M. B. Lopes dos Santos, A. H. Castro Neto, A. Reina, J. Kong, E. Y. Andrei, Nature Phys. 6 (2010) 109.

[16] G. Li, A. Luican, E. Y. Andrei, Phys. Rev. Lett. 102 (2009) 176804.

[17] A. Luican, G. Li, A. Reina, J. Kong, R. R. Nair, K. S. Novoselov, A. K. Geim, E. Y. Andrei, Phys. Rev. Lett. 106 (2011) 126802.

[18] W. Yan, M. Liu, R. F. Dou, L. Meng, L. Feng, Z. D. Chu, Y. Zhang, Z. Liu, J. C. Nie, L. He, Phys. Rev. Lett. 109 (2012) 126801.

[19] P. Poncharal, A. Ayari, T. Michel, J. L. Sauvajol, Phys. Rev. B 79 (2009) 195417. 
[20] Z. Ni, L. Liu, Y. Wang, Z. Zheng, L. J. Li, T. Yu, Z. Shen, Phys. Rev. B 80 (2009) 125404.

[21] K. Kim, S. Coh, L. Z. Tan, W. Regan, J. M. Yuk, E. Chatterjee, M. F. Crommie, M. L. Cohen, S. G. Louie, A. Zettl, Phys. Rev. Lett. 108 (2012) 246103.

[22] R. W. Havener, H. Zhuang, L. Brown, R. G. Hennig, J. Park, Nano Lett. 12 (2012) 3162.

[23] V. Carozo, C. M. Almeida, E. H. M. Ferreira, L. G. Cancado, C. A. Achete, A. Jorio, Nano Lett. 11 (2011) 4527.

[24] J. H. Warner, M. H. Rümmeli, T. Gemming, B. Büchner, G. A. D. Briggs, Nano Lett. 9 (2009) 102.

[25] L. Brown, R. Hovden, P. Huang, M. Wojcik, D. A. Muller, J. Park, Nano Lett. 12 (2012) 1609 .

[26] J. T. Robinson, S. W. Schmucker, C. B. Diaconescu, J. P. Long, J. C. Culbertson, T. Ohta, A. L. Friedman, T. E. Beechem, ACS Nano 7 (2013) 637.

[27] H. Schmidt, T. Lüdtke, P. Barthold, E. McCann, V. I. Fal'ko, R. J. Haug, Appl. Phys. Lett. 93 (2008) 172108.

[28] H. Schmidt, T. Lüdtke, P. Barthold, R. J. Haug, Phys. Rev. B 81 (2010) 121403(R).

[29] D. S. Lee, C. Riedl, T. Beringer, A. H. Castro Neto, K. von Klitzing, U. Starke, J. H. Smet, Phys. Rev. Lett. 107 (2011) 216602.

[30] J. D. Sanchez-Yamagishi, T. Taychatanapat, K. Watanabe, T. Taniguchi, A. Yacoby, P. Jarillo-Herrero, Phys. Rev. Lett. 108 (2012) 076601.

[31] B. Fallahazad, Y. Hao, K. Lee, S. Kim, R. S. Ruoff, E. Tutuc, Phys. Rev. B 85 (2012) 201408(R).

[32] Y. Kim, H. Yun, S. -G. Nam, M. Son, D. S. Lee, D. C. Kim, S. Seo, H. C. Choi, H. -J. Lee, S. W. Lee, J. S. Kim, Phys. Rev. Lett. 110 (2012) 096602.

[33] D. H. Chae, D. Zhang, X. Huang, K. von Klitzing, Nano Lett. 12 (2012) 3905.

[34] L. A. Ponomarenko, R. V. Gorbachev, G. L. Yu, D. C. Elias, R. Jalil, A. A. Patel, A. Mishchenko, A. S. Mayorov, C. R. Woods, J. R. Wallbank, M. Mucha-Kruczynski, B. A. Piot, M. Potemski, I. V. Grigorieva, K. S. Novoselov, F. Guinea, V. I. Fal'ko, A. K. Geim, Nature 497 (2013) 594.

[35] C. R. Dean, L. Wang, P. Maher, C. Forsythe, F. Ghahari, Y. Gao, J. Katoch, M. Ishigami, P. Moon, M. Koshino, T. Taniguchi, K. Watanabe, K. L. Shepard, J. Hone, P. Kim, Nature 497 (2013) 598.

[36] B. Hunt, J. D. Sanchez-Yamagishi, A. F. Young, M. Yankowitz, B. J. LeRoy, K. Watanabe, T. Taniguchi, P. Moon, M. Koshino, P. Jarillo-Herrero, R. C. Ashoori, Science 340 (2013) 1427. 
[37] A. W. Tsen, L. Brown, M. P. Levendorf, F. Ghahari, P. Y. Huang, R. W. Havener, C. S. RuizVargas, D. A. Muller, P. Kim, J. Park, Science 336 (2012) 1143.

[38] Z. Luo, Y. Lu, D. W. Singer, M. E. Berck, L. A. Somers, B. R. Goldsmith, A. T. C. Johnson, Chem. Mater. 23 (2011) 1441.

[39] A. Reina, H. Son, L. Jiao, B. Fan, M. S. Dresselhaus, Z. Liu, J. Kong, J. Phys. Chem. C 112 (2008) 17741.

[40] P. Y. Huang, C. S. Ruiz-Vargas, A. M. van der Zande, W. S. Whitney, M. P. Levendorf, J. W. Kevek, S. Garg, J. S. Alden, C. J. Hustedt, Y. Zhu, J. Park, P. L. McEuen, D. A. Muller, Nature 469 (2011) 389.

[41] H. Cao, Q. Yu, L. A. Jauregui, J. Tian, W. Wu, Z. Liu, R. Jalilian, D. K. Benjamin, Z. Jiang, J. Bao, S. S. Pei, Y. P. Chen, Appl. Phys. Lett. 96 (2010) 122106.

[42] I. Skachko, X. Du, F. Duerr, A. Luican, D. A. Abanin, L. S. Levitov, E. Y. Andrei, Phil. Trans. R. Soc. A 368 (2010) 5403.

[43] N. Kim, K. S. Kim, N. Jung, L. Brus, P. Kim, Nano Lett. 11 (2011) 860.

[44] A. N. Ramanayaka, R. G. Mani, Phys. Rev. B 82 (2010) 165327.

[45] K. S. Novoselov, E. McCann, S. V. Morozov, V. I. Fal'ko, M. I. Katsnelson, U. Zeitler, D. Jiang, F. Schedin, A. K. Geim, Nature Phys. 2 (2006) 177.

[46] D. A. Abanin, L. S. Levitov, Phys. Rev. B 78 (2008) 035416.

[47] M. Monteverde, C. Ojeda-Aristizabal, R. Weil, K. Bennaceur, M. Ferrier, S. Guéron, C. Glattli, H. Bouchiat, J. N. Fuchs, D. L. Maslov, Phys. Rev. Lett. 104 (2010) 126801.

[48] R. de Gail, M. O. Goerbig, F. Guinea, G. Montambaux, A. H. Castro Neto, Phys. Rev. B 84 (2011) 045436.

[49] M. Y. Choi, Y. H. Hyun, Y. Kim, Phys. Rev. B 84 (2011) 195437. 


\section{Figure caption}

Fig. 1. (a) Fabrication process of tBLG device on $\operatorname{SiN}_{\mathrm{x}}$ substrate. (b) Optical (left) and TEM (right) image of the device. Scale bar in optical image is $20 \mu \mathrm{m}$. TEM image indicates red dashed line in optical image and shows two-probe measurement scheme. The tBLG channel is guided by blue rectangle.

Fig. 2. (a) Raw data of Raman spectrum of tBLG with twist angle, $2^{\circ}$. (b) Raman spectroscopy of two different twist angle of tBLG after subtracting background substrate signal. $(\mathrm{c}, \mathrm{d})$ Electron diffraction patterns of tBLG with twist angle, $2^{\circ}$ and $18^{\circ}$, respectively.

Fig. 3. (a) Raw data of R vs $B$ of tBLG with twist angle, $2^{\circ}$ at several low temperatures. (b) $\Delta \mathrm{R}$ vs $B$. (c) Landau fan diagram of $2^{\circ}$ angle at lowest temperature, $2.8 \mathrm{~K}$. Lower inset indicates Fourier transform of $\Delta \mathrm{R}\left(=\mathrm{R}-\mathrm{R}_{\text {background }}\right)$ vs $1 / B$ plot. Upper inset shows superimposed oscillation enlarged in the red dashed box. Blue circles indicate $n(n+1 / 2)$-th LL corresponding to left axis and orange lines mean linear fitting of the Landau fan diagram. (d) Landau fan diagram of superimposed oscillation after subtracting the slow oscillation part in the upper inset of Fig. 3(c).

Fig. 4. (a) Raw data of R vs $B$ of tBLG with twist angle, $18^{\circ}$ at several low temperatures. (b) $\Delta \mathrm{R}$ vs $B$. (c) Landau fan diagram of $18^{\circ}$ angle at lowest temperature, $2.8 \mathrm{~K}$. Lower inset indicates Fourier transform of $\Delta \mathrm{R}\left(=\mathrm{R}-\mathrm{R}_{\text {background }}\right.$ ) vs $1 / B$ plot. Upper inset shows superimposed oscillation enlarged in the red dashed box. (d) Landau fan diagram of superimposed oscillation after subtracting the slow oscillation part in the upper inset of Fig. 4(c). Data analysis process is same with $2^{\circ}$ angle case as shown in Fig. 3 .

Fig. 5. Summary of intercept values of $2^{\circ}$ and $18^{\circ}$ angles. While intercept values are close to 0.5 regardless of carrier density in $18^{\circ}$ angle, the values depend on carrier density in $2^{\circ}$ angle. High carrier density yields nearly 0 intercept value similar with that of Bernal stacked BLG.

Fig. 6. (a) Energy band plot of large twist angle case. (b) Band plot with interlayer potential $\Delta_{\mathrm{z}}$ (dashed red curve) and the interlayer coupling with strength $\Gamma$ (solid black curve). (c) Contour plot of Fermi surface of large twist case. Black circles indicate the Fermi level with chemical potential represented by blue dashed line in (b). (d)-(e) Same approaches with (a)-(b) for small twist case. (f) Contour plot of small twist case with chemical potential comparable to (c). 
Fig. 1.

(a)

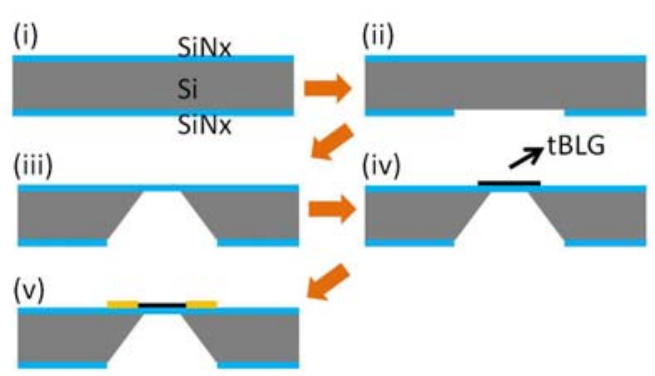

(b)

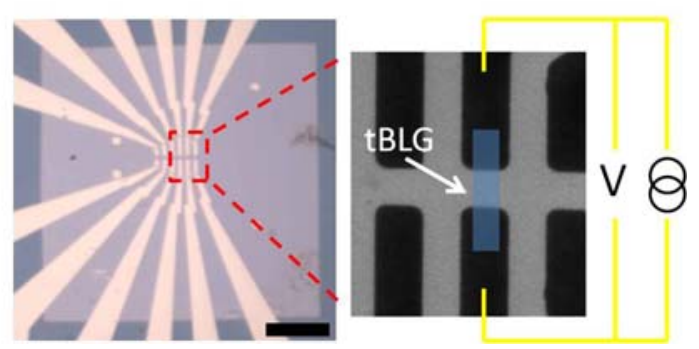


Fig. 2.
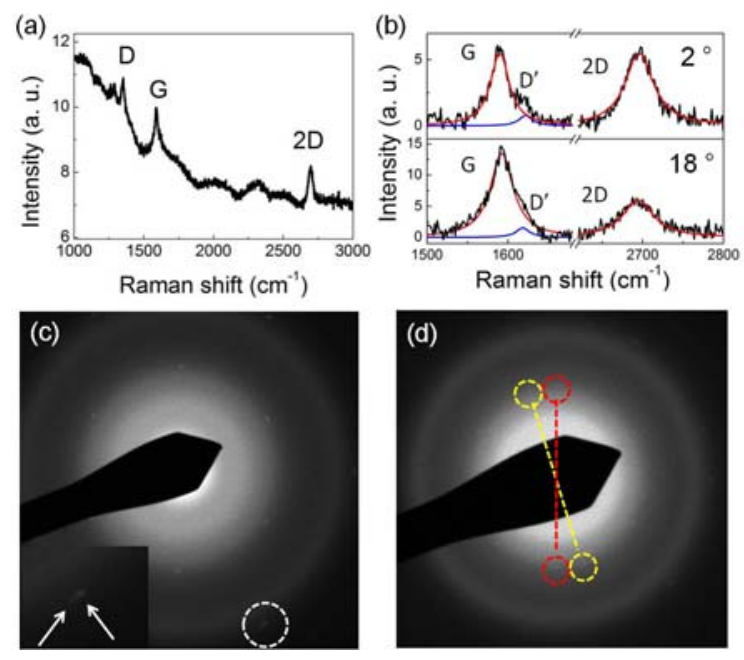
Fig. 3.
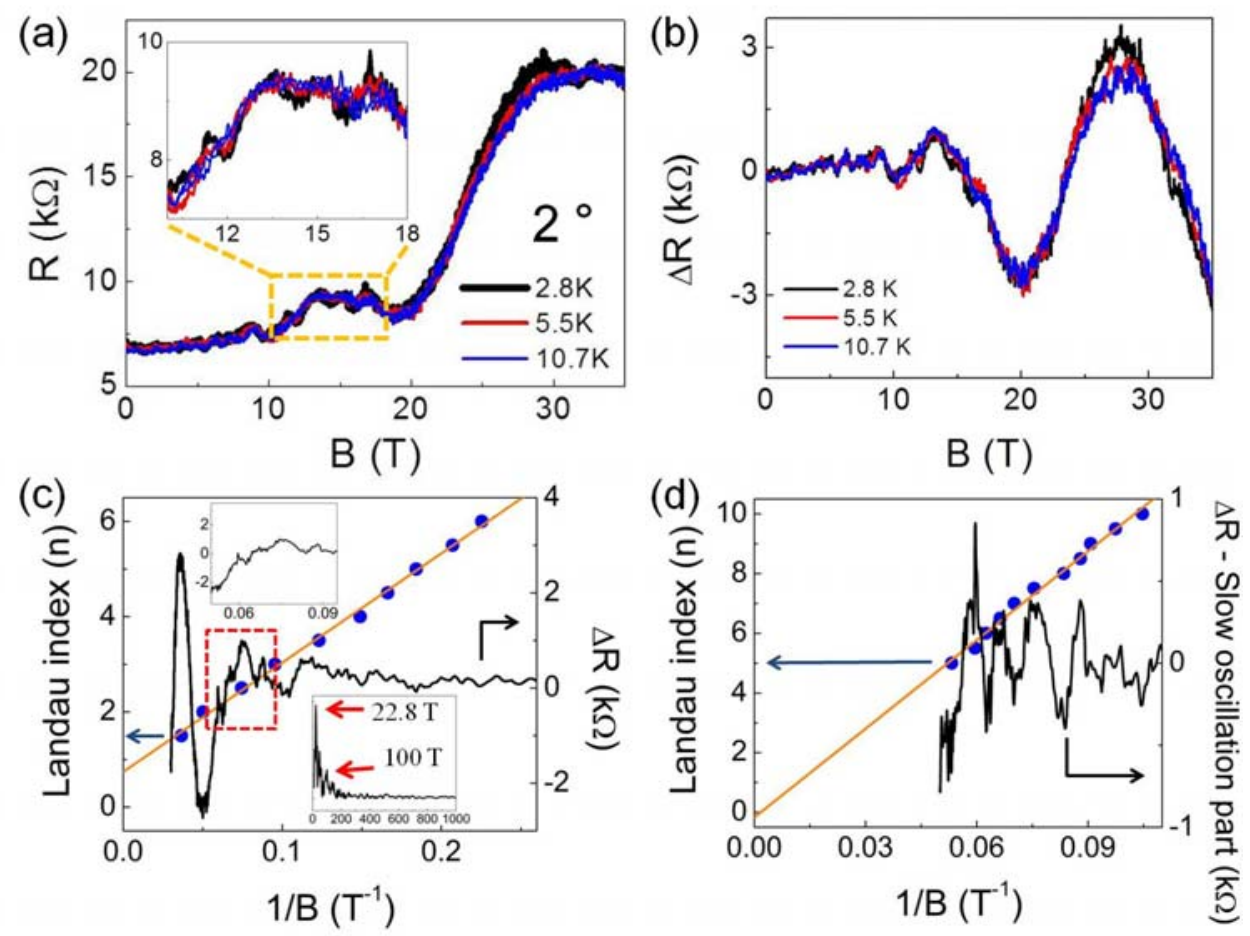
Fig. 4.
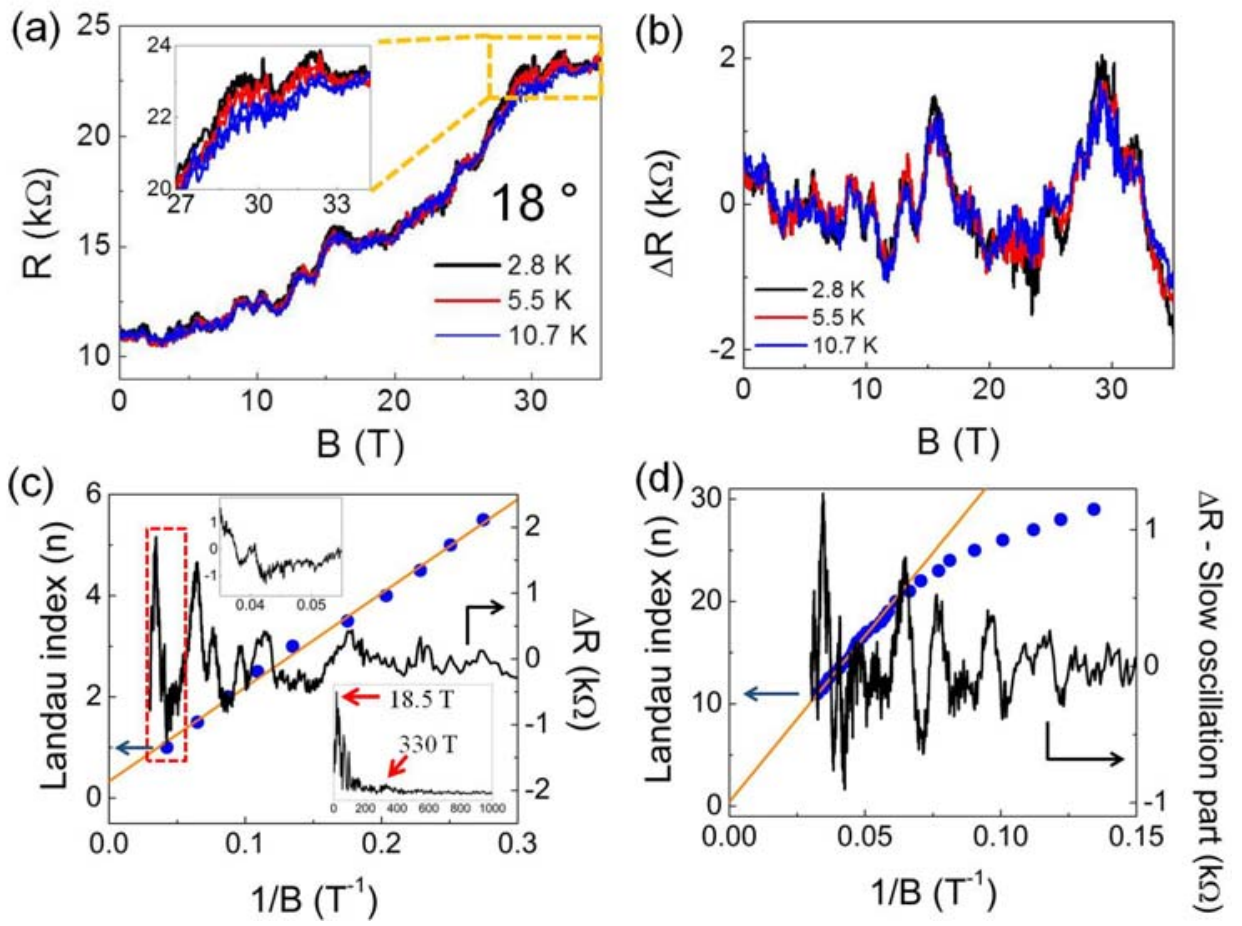
Fig. 5.

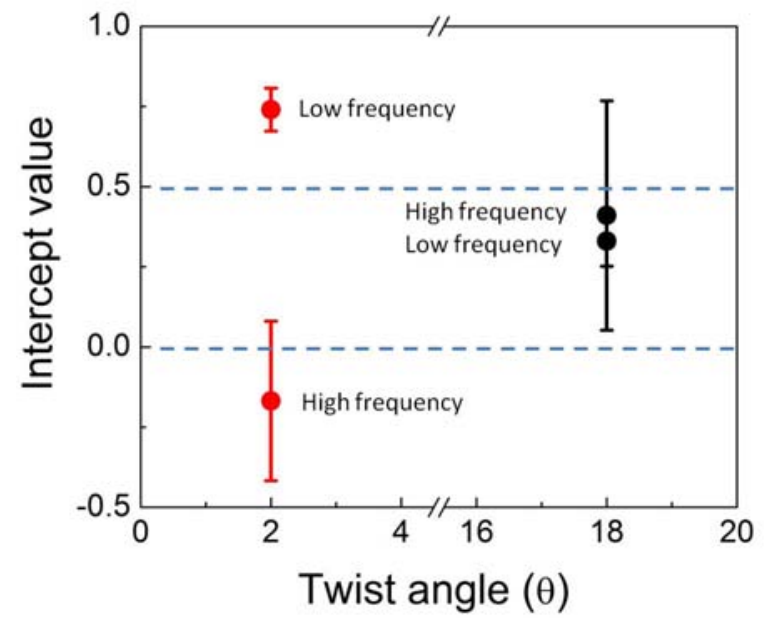


Fig. 6.
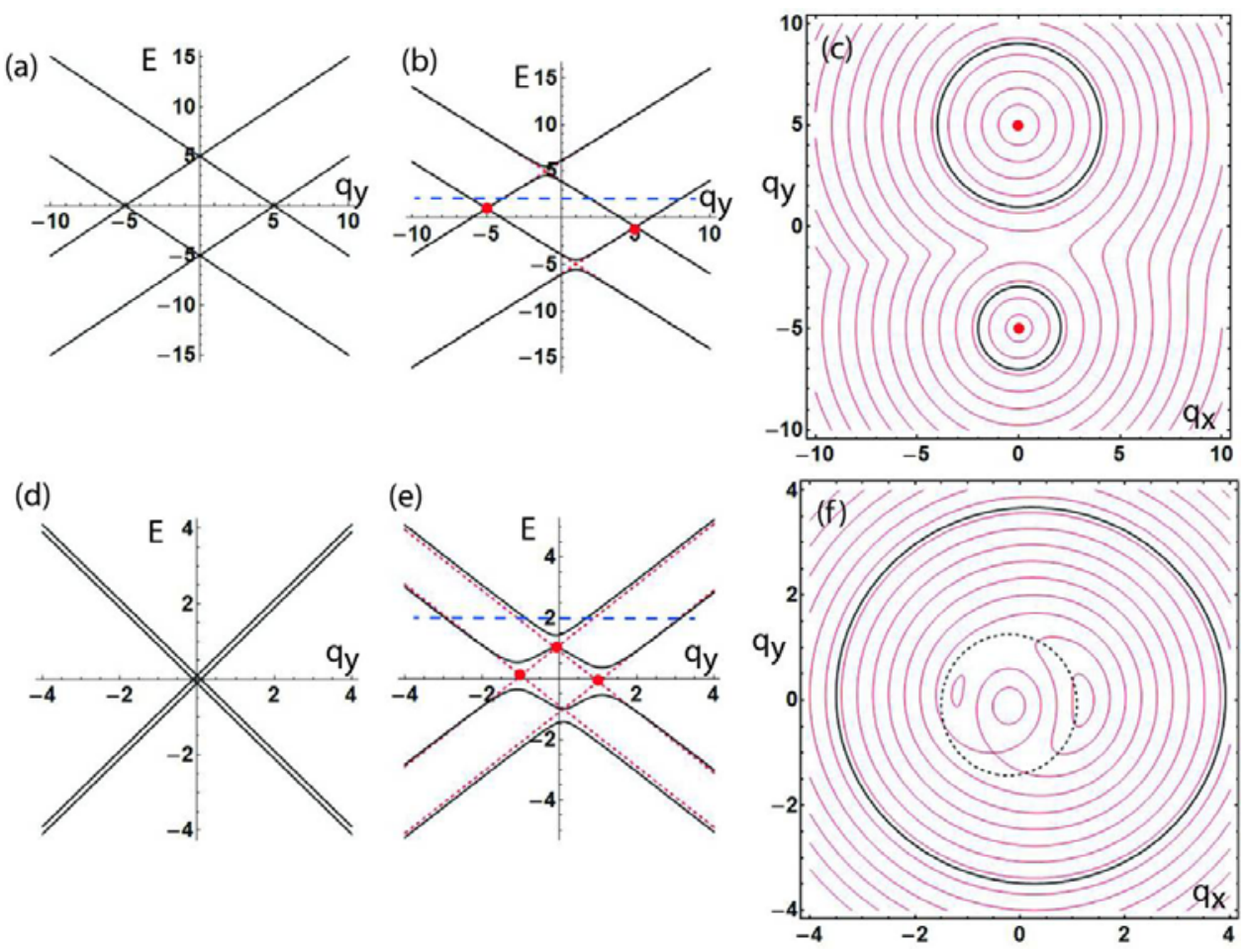

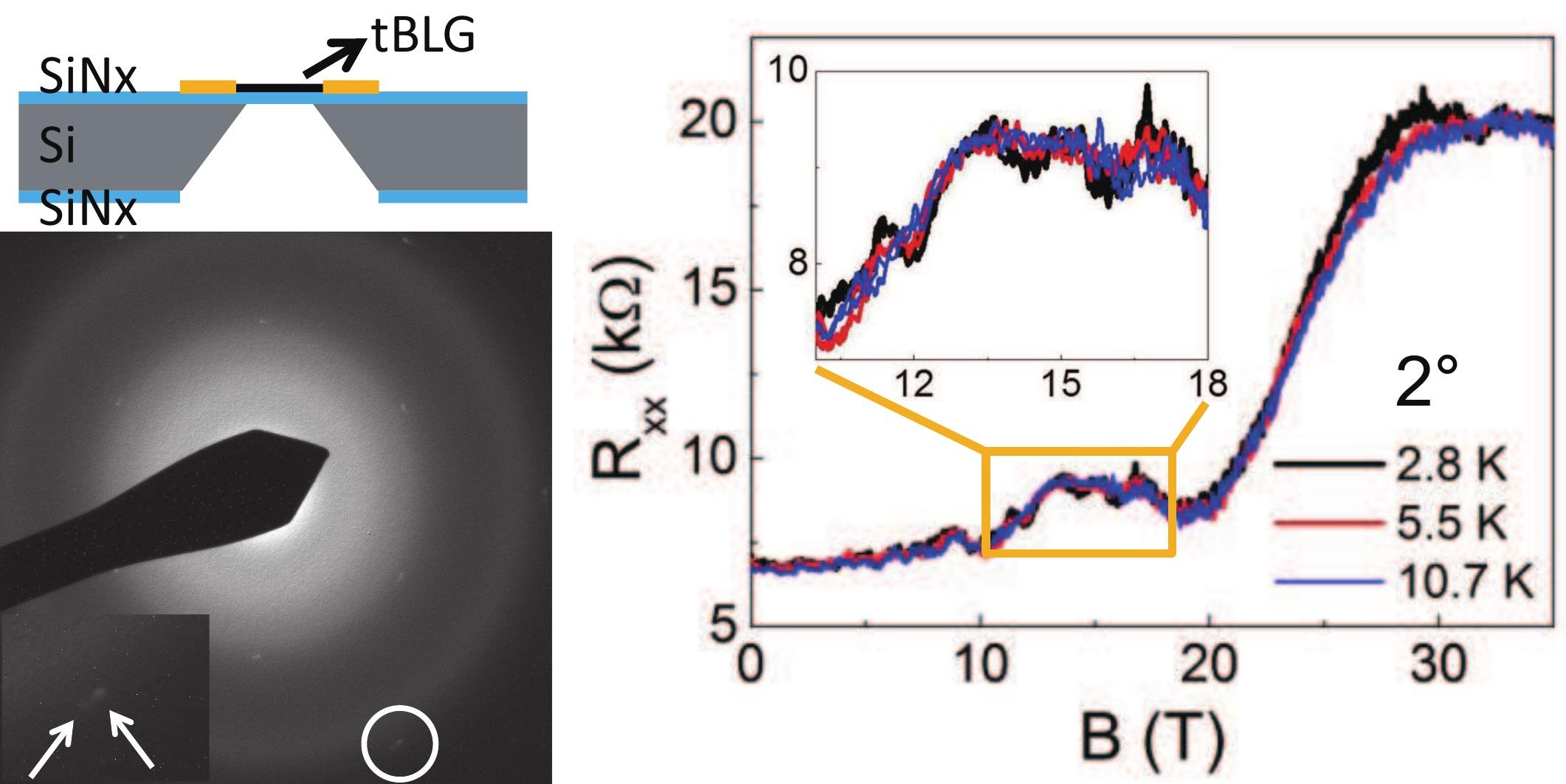